\title{
An Online Participatory Observation: The Online Depression Community's Discussion on Depression Treatment
}

\author{
Wu, Xinzhu \\ College of Communication, National Chengchi University, \\ Taipei City 11605,China (P.R.C); \\ Email: hsinzhu98 [AT] gmail.com
}

\begin{abstract}
This article uses the online participatory observation method to analyze more than 2,000 posts of the "Sunshine Project Psychological Mutual Assistance Forum" in Mainland China. The research observed the discussion of depression in the online patient community in anonymous forums, including the popularization of medical knowledge about diseases, self-disclosure of patient diagnosis and treatment experience, patient interaction, and patient discussions on controversial issues and rumors of the disease. The results of the study show that the online community of depression patients is both a "web of information" and a "web of mutual help." The patient's health information acquisition mode has changed from vertical reception to horizontal interconnection, which not only enhances patients' scientific understanding of the disease but also allows them to increase their sense of psychological security in their interpersonal interactions. In this new type of risk communication mode, how to establish a new fact-checking mechanism and establish ordinary people's trust in scientific and medical knowledge is a new challenge.
\end{abstract}

Keywords - health communication, depression, science communication, depression community, online participation observation

\section{INTRODUCTION}

The latest research report of the World Health Organization shows that about 350 million people worldwide are suffering from depression. In 2020, among the top ten diseases that cause human disability, depression ranks first. Up to 1 million people die by suicide due to depression each year. During the COVID-19 period, the number of patients with mental illness has increased rapidly(WHO, 2020). There is no doubt that depression has become a problem that cannot be underestimated.

Although the medical profession already has effective treatments for depression, survey data show that less than half of the patients worldwide receive effective treatment (and even less than 10\% in some countries) (WHO, 2020). The medical knowledge of and depression treatment is not universal in many countries and regions. In addition, many empirical studies point out social discrimination and stigmatization of mental illness may be an important reason why patients are unwilling to receive formal treatment (Zhong Nanshan, 2008; WHO, 2020).

Reviewing past studies on depression from the perspective of health communication, the author finds that scholars pay more attention to the reappearance of depression in the mass media. There is less research on the cognition, attitude, and self-disclosure of the depression community. How the depression community views depression treatment, what sources of information are based on their cognition, how they respond to and discuss the controversial issues in depression treatment, are the issues that this paper cares about.

With the acceleration of digitization, popular medical science applications and forums have also entered the public eye. The audience is no longer just passively and unconsciously receiving health news from newspapers and magazines, but on the Internet and communities, actively searching and obtaining medical information, seeking horizontal interconnection, sharing and exchanging health information. At the same time, however, the barriers to entry for the media have been lowered, and the explosive growth of information has also brought new difficulties: health information is uneven, medical "fake news" has sprung up like mushrooms, and rumors and panics have accelerated.

This research attempts to discuss how in the era of digital interconnection, scientific knowledge of diseases is disseminated, and how non-scientific stereotypes are reshaped? What dawns and challenges does the sharing of patient information bring to health communication? In the post-epidemic era, where should the communication on health and 
risk issues go?

So far, the diagnosis and treatment of depression are still in the form of consultation. It means that the diagnosis and cure of depression do not have General physiological indicators (such as blood pressure, indicator concentration, etc.) like other physiological diseases. Due to the particularity of the diagnostic criteria and pathological characteristics of mental illness, patients' cognition of depression and depression treatment is not only influenced by general scientific and biomedical knowledge, but also by their own disease experience to a large extent. Therefore, observing how the depression community discusses the treatment of depression can also give a glimpse of the balance and contradiction between the subjective experience of such a mental illness community and the biomedical reality of the disease. And in this context, how does science communication affect their cognition, and what efforts can be made.

This study adopts the online participatory observation method to sort out and review the public posts proactively released by the depression community on the "Sunshine Engineering Psychological Mutual Assistance Forum" and explore how the depression community discusses the treatment of depression.

\section{DEPRESSION AND DEPRESSION COMMUNITY}

Depression is often closely linked to emotions, personality, and philosophy. Pathological depression is slightly different from melancholy. The definition of depression in clinical medical research focuses more on its classification, diagnostic symptoms, etiology, and treatment. The "Diagnostic and Statistic Manual Mental Disorders, 4th edition, DSM-IV" published by the American College of Psychiatrists divides depression into mild, moderate, severe depression, and low-grade affective disorders. Depression is widely considered pathologically as the result of a complex interaction of social, psychological, and physiological factors (WHO, 2020). In clinical medicine, the causes of depression can be divided into "biological risk factors", such as family genetic factors, the lack of monoamine neurotransmitters and the physiological mechanism of physical diseases, and "psychological-social risk factors", as Environmental factors, lost incidents and insufficient social support in 2015 (National Taiwan University School of Medicine, 2018). Medical research has pointed out that hyperactivity of the hypothalamus-pituitary gland-adrenal cortex (HPA) system in patients with depression is the cause of anxiety, decreased libido and appetite, and sleep disorders (Stansbury \& Gunnar, 1994).

So far, the diagnosis and treatment of depression still mainly rely on the form of consultation, and the diagnosis of depression still relies on the judgment of symptoms by psychiatrists with professional experience. Clinically, the "Diagnostic Manual of Mental Disorders (Fifth Edition)" defines depression as: an individual has experienced at least two weeks or more and has at least the following five symptoms almost every day, and includes (1) or (2): (1) Mood Low; (2) Obvious loss of interest in things; (3) Weight loss or gain; (4) Insomnia or drowsiness; (5) Sluggishness or increased activity due to restlessness; (6) Tiredness or loss of vitality; (7) Conscious sense of worthlessness or guilt; (8) decreased thinking ability or concentration; (9) suicidal ideation, etc. (American Psychiatric Association, 2013).

According to the latest research report of the World Health Organization (WHO), there are 350 million people suffering from depression worldwide, and the annual suicide death toll is estimated to be as high as 1 million. Suicide is the second leading cause of death in the 15-29 age group (WHO, 2020).

Data show that as of 2019, there are more than 95 million people suffering from depression in China. Among them, adolescent depression is more than $50 \%$, and high school students account for $40 \%$. Statistics released by the World Health Organization (WHO) show that nearly half of global depression patients live in Southeast Asia and the Western Pacific, including India and China (WHO, 2019). In 2019, the prevalence data report of the China Health Survey (CMHS) showed that the lifetime prevalence of depression in China was 6.9\%. Survey data on depression in Chinese adults show that Shaanxi, Gansu, Fujian, and other regions have the highest proportion of patients with severe depression. An 18-year study of Chinese college students including more than 30,000 subjects showed that the incidence of depression among Chinese students was 23.8\% (Medsci, 2019). According to the "Guidelines for the Prevention and Treatment of Depressive Disorders in China", the overall recurrence rate of major depressive disorder is as high as $50 \%-$ $85 \%$, and $50 \%$ of patients relapse within two years after the onset of the disease.

The depression community that this research focuses on is those who actively post posts in the depression public forum, are diagnosed or self-identified as depression patients, or actively talk about depression-related experiences or information. The depression community includes not only depression patients, relatives or friends of depression patients, depression treatment physicians, or people who are not depressed but self-identify as having depression or depression tendencies.

\section{THE BIOMEDICAL REALITY OF DEPRESSION TREATMENT}

The current medical profession believes that depression must be carefully diagnosed by a physician before the symptoms and medication can be determined (Chen Junqin, 2004). The drug treatment of depression originated in the early 1950s. The inhibitory effect of monoamine oxidase (MAO) was found in the structure of the anti-tuberculosis drug Isoniazid and other drugs. After taking such drugs, patients showed emotional activity, insomnia, and excitement. These 
drugs are called monoamine oxidase inhibitors (MAOs), which were first used to treat depression. Subsequently, tricyclic and tetracyclic drugs were gradually developed, which reduced the side effects of MAOs. In the 1980s, fluoxetine was marketed in the United States. It was called a serotonin reuptake inhibitor (SSRIsk) with paroxetine, sertraline, fluvoxamine, citalopram, and other drugs. The advantages are more obvious, and the side effects are also more obvious. Even more minor, it is still a common first-line treatment for depression currently on the market (Zhang Jiexia, 2010; China Blue Book of Depression, 2019).

The biochemical factors in the cause of depression cannot be ignored, and some studies believe that drugs that promote the secretion of neurotransmitters may be effective for $70 \%$ of patients. Therefore, the current research and development of antidepressant drugs in the medical field is mostly related to trying to promote the secretion of neurotransmitters (Ye Sujun, 2010). Antidepressants can relatively effectively relieve the anxiety, tension, and physical symptoms associated with depressive moods, with an effective rate of about $60 \%$ to $80 \%$ (Blue Book of the Field of Depression in China, 2019).

Psychotherapy for depression can be used alone for patients with mild depression. Common therapies include cognitive behavior therapy (CBT), interpersonal psychotherapy (IPT), dialectical behavior therapy (DBT), mindfulness therapy, psychodynamic therapy, and human Capitalist treatment. It also includes sports, reading, art therapy, etc.

The most widely used is individual psychotherapy in the treatment of depression, which doctors based on general common sense and understanding of human nature, through the establishment of a good doctor-patient relationship, effective communication, and psychological counseling (Shu Manqiao, 2014).

Among the special psychological treatments for depression, the more common one is interpersonal psychotherapy. In the 1960s, Weissmen and Klerman's research found that many people with depression have interpersonal problems. If interpersonal problems are improved, the symptoms of depression will also be reduced. As a result, Interpersonal Psychotherapy has received widespread attention as a special psychological treatment method for depression. Interpersonal psychotherapy refers to the treatment of depression through a short-term limited time individual or group treatment, supplemented by drugs (Tang Zijun, 2018). The NIMH Treatment of Depression Collaborative Research Program conducted large-scale comparisons of various depression treatments and tried different combinations of treatments, and found that interpersonal psychotherapy is quite effective among many therapies. Compared with the effect of general psychotherapy about half a year, the long-term therapeutic effect of interpersonal psychotherapy can last up to two years.

Another more common form of psychotherapy is cognitive behavioral therapy. Baker believes that people often use the structure and mode of interpretation and thinking that they have accumulated from experience to understand and solve problems in life, and the individual may not be aware of it. It is a kind of "automatic thoughts" ( Lin, 2001). Cognitive behavioral therapy emphasizes the learning process, that is, learning new ways of thinking in the process of consultation, changing one's original bad thinking style, and then solving distressing emotions (Chen Caihui, 2004).

The treatment of depression mainly depends on medication and psychotherapy. However, about $20 \%$ of patients expressed poor efficacy during long-term follow-up visits. Physiotherapy has also been used in the treatment of clinical depression (Blue Book of the Field of Depression in China, 2019).

Physiotherapy for depression mainly includes electroconvulsive therapy, non-convulsive electroconvulsive therapy, and transcranial magnetic therapy. Electro-convulsive therapy means that electric current is passed to the patient's brain to trigger the self-discharge of the patient's brain complement cells (Synchronized Discharge) so that the patient's brain function can be adjusted (Liu Yi'an, 2017). The history of electrotherapy can be traced back to the 1930s. In 1938, the first patient with schizophrenia who received electrotherapy appeared. After eleven electrotherapies, the patient fully recovered. Scientists discovered that spasm can be used to treat mental illness (Abrams R, 2002).

The depression community or ordinary people's fear of electrotherapy may stem from the implementation process of electroshock therapy and its side effects. In this regard, data from the National Center for Biotechnology shows that electric shock therapy has sequelae such as short-term memory impairment, headache, nausea, and vomiting, or general muscle aches, but these sequelae can be relieved by taking medicine after the course of treatment. The data also pointed out that no treatment is completely free of sequelae, and the sequelae of electroshock therapy are less than drug therapy.

Convulsion-free electroconvulsive therapy is an improved electrical convulsive therapy, and transcranial magnetic therapy is a painless and non-invasive green treatment method that stimulates the brain through low-intensity trace currents. The principle is similar to that of electrical convulsions.

With the advancement of technology and medical and health technology, other treatments for depression, including new technologies such as the use of artificial intelligence technology to determine depression using voice and visual recognition systems, are also in the stage of $R \& D$ and clinical scientific research cooperation. Since this therapy has not been widely used, it is not within the scope of this article's observation and research. 
In the medical field, although depression has developed a series of treatments. However, studies have pointed out that only $35 \%$ of patients can get symptom improvement in the end, and $65 \%$ of patients still suffer from depression (Lin, 2001).

For the treatment of diseases, people often use "treatable" and "curable" to define. And whether depression can be completely cured, there is still no definite conclusion in the medical field. It is also difficult for doctors of depression to make a diagnosis that the disease is completely cured because depression is very easy to relapse. Data shows that a patient with depression may face 4-8 relapses in his life.

\section{THE MEDIA REALITY AND SOCIAL CONSTRUCTION OF DEPRESSION COMMUNITY}

Looking at the communication research in the field of mental health at home and abroad, it can be found that the media's reappearance of mental illness is mostly presented in a negative form (Klin \& Lemish, 2008; Morris, 2006; Nairn, 2007; Philo, 1996; Wahl \& Roth, 1982; Wahl, Wood, \& Richards, 2002; Wu Mengjin, Xu Meiling, 2011). A series of empirical studies on the topic of depression show that the mass media in mainland China has always had the problem of lack of normalized attention and improper reporting strategies in reporting depression. The research conducted by mainland Chinese scholar Dong Wei (2010) observed that depression reports in the Health and Sanitation News, popular newspapers, and party newspapers from 2000 to 2008 shaped the identity of patients with depression, and found that the mainstream media lacked attention to depression, and the media reported relatively Violent and negative. Wang Cuitong's research also pointed out that the image of depression patients in newspapers deviates from the image in real life.

In 2016, Pang Xu observed the depression reports in four newspapers of different nature from 2011 to 2015 . The results of the study show that although depression has aroused widespread concern and discussion by then, the number of reports on depression and its patients in newspapers is still flat, and there is no obvious increasing trend. Moreover, in the understanding of depression treatment, the media did not emphasize the necessity and importance of professional treatment and even had a biased and contemptuous attitude in the definition of the problem. In addition, the analysis of TV and online media also pointed out the same stigma problem (Ren Jinzhou, Kang Yunkai, 2015).

In 2018, Jiang Yang analyzed depression-related posts on social media and pointed out that mainstream media's discussion of depression is event-oriented and lacks a discussion on prevention and treatment. The problem definition is still vague, and the phenomenon of negative evaluation also exists. The research also found through the analysis of audience comments that the audience's cognitive ability on public health issues is not enough, and there is a deviation in the understanding of diseases.

Coincidentally, the media reproduction research of Wu Mengjin and Xu Meiling (2011) pointed out that the reports of depression in Taiwan also have the problem of insufficient medical knowledge and information on prevention and treatment. However, the study pointed out that the stigmatization of depression reports in Taiwanese media has improved.

Looking at the academic research in the field of Chinese health communication, there is relatively little attention to depression. Scholars in the field of journalism pay less attention to issues of mental illness such as depression than AIDS and diabetes (Jiang Yang, 2018). The research on depression mostly adopts media observation and content analysis methods, and most of the research samples are concentrated in traditional media such as newspapers and television. With the rise of social networks, scholars have gradually turned their horizons to social platforms such as Weibo. However, previous studies mostly focused on the analysis of news texts and mainstream media. Internet research also mainly focused on the publications and posts of mainstream media public accounts. There are few studies on the self-disclosure and cognitive attitudes of patients with depression. Due to the particularity of its mental illness, the patient's own experience and sense of illness are important factors that affect the choice of treatment for patients with depression, which deserves attention and research.

\section{RESEARCH METHOD}

This study uses the Online Participant Observation method. The research crawls through the posts related to treatment and medication topics published by the depression community in the Sunshine Psychological Mutual Assistance Forum (www.sunofus.org). The Sunshine Project Psychological Mutual Assistance Forum was set up by the "Sunshine Project Love Action", China's first charity organization to combat depression. It is committed to providing melancholic knowledge and information, providing a platform for exchanges and mutual assistance for patients with depression, and sharing rehabilitation experiences. There are more than 900,000 posts on this website, which not only provide posting, commenting, and retrieval functions, but also detailed classification according to "essays", "help information", "medical information" and other partitions, so it basically meets the observation needs of this research. 
In response to the questions raised in this research, after screening, the author observed a total of 30 posts and 2,211 comments after the posts. These 30 posts are the self-disclosure of the depression community's perceptions, attitudes, diagnosis and treatment experience of various treatments for depression, and discussions on controversial issues. The author also observes the diachronic changes in the discussion of patients with depression.

\section{RESEARCH RESULTS}

\subsection{The community that talks about depression in online forums}

Crawling through the online posts, it can be found that the groups discussing depression in public online forums can be roughly divided into the following categories: people who are diag nosed with depression, those who self-identify as depression, ordinary people with depression tendency, and family/friends. Ordinary people who are diagnosed with depression, doctors who specialize in mental or psychiatric diseases such as depression, and general netizens who are interested in the topic of depression. As shown in the following table:

Table 1: Depression community and their posts content

\begin{tabular}{|c|c|}
\hline Depression community & Posts content \\
\hline $\begin{array}{l}\text { Patients diagnosed with depression } \\
\text { People who self-identified as melancholic }\end{array}$ & $\begin{array}{l}\text { essay logs focusing on talking or recording mood, } \\
\text { self-disclosure of disease characteristics, sharing and } \\
\text { exchange of rehabilitation experience, consultation, } \\
\text { and mutual assistance of medical information }\end{array}$ \\
\hline $\begin{array}{l}\text { Ordinary people with melancholic } \\
\text { tendencies }\end{array}$ & $\begin{array}{l}\text { Reveal their self-characteristics and confirm the } \\
\text { criteria for the diagnosis of melancholia }\end{array}$ \\
\hline $\begin{array}{c}\text { Relatives/friends are ordinary people who } \\
\text { suffer from depression }\end{array}$ & $\begin{array}{l}\text { Reporting of patient characteristics and seeking help } \\
\text { for solutions }\end{array}$ \\
\hline $\begin{array}{c}\text { Physician who specializes in depression or } \\
\text { mental illness }\end{array}$ & $\begin{array}{l}\text { rPopularization of treatment knowledge, interpretation } \\
\text { of controversial issues, clarification of rumors }\end{array}$ \\
\hline $\begin{array}{c}\text { General netizens who are interested in the } \\
\text { topic of depression }\end{array}$ & $\begin{array}{l}\text { Investigating and asking questions about depression } \\
\text { experience, and sharing the latest treatments }\end{array}$ \\
\hline
\end{tabular}

In the public forums, the main content of posts by people who have been diagnosed with depression and those who self-identify as depression can be roughly divided into essay logs focusing on talking or recording mood, self-disclosure of disease characteristics, and sharing or exchange of rehabilitation experience, Medical information consultation and mutual assistance. The above several types of posts also overlap with each other. Essay diaries that focus on recording or confiding emotions are often short in length, and the narrative logic is relatively fragmented, and the sentences are mostly short sentences to vent the mood. However, follow-up responses from the original poster (post publisher) are more frequent. For example, in 2015, the netizen "S" posted "Record Your Feelings":

There is a sense of ease in lying in bed that you don't need to do anything, and it feels uncomfortable to do so, and you want to do something but don't want to do it, as if you shouldn't be comfortable.

The host of this post made another 83 replies to the post within two years after the first post. Among them are the responses to the comfort and encouragement of netizens, the intermittent records of their own life and illness experience, and their mood.

The post on the theme of sharing and introducing rehabilitation experience is longer, with a more complete narrative logic and structure. For example, the netizen "M"'s post "[Severe Depression Rehabilitation Experience] [Western Medicine, Running, Chinese Medicine, Cognition, Religion] [Suppressing Friends' Hope] Three Fits One" published about 7,000 words of illness and illness for the first time The sharing of rehabilitation experience described in detail his characteristics during the period of illness and his experience in trying various therapies. In such posts, in addition to sharing their own recovery experience, patients usually make suggestions to patients who are still struggling with depression as recovered people. The most obvious feature is the extensive use of the second person.

Such as the post of the netizen "M":

It is an objective fact that you came into this world. You have to learn to accept it and learn to survive bravely. Life and death are both objective and not shifted by your subjective consciousness, so there is no need to think too much. 
Another example is the post "[Original] My Self-Healing Feelings" by netizen "Y":

If you simply endure pain and admit that it is an inescapable pain, two or three hours will pass. If you hope to eliminate it by talking, you may still be talking today next year. This is not alarmist.

The general netizens with depressive tendencies usually post in the form of self-disclosure and information on depression standards. Most of his articles are "I have XXX symptoms, is this depression? ". Such as "Am I Really Depressed?" ", "Emotional instability, is this depression? ". Such posts usually simply describe their own situation and ask whether their symptoms meet the diagnostic criteria for depression. It is worth mentioning that some netizens with depressive tendencies may also seek ways to change their depression, anxiety, and other depressive emotions in their posts, but the word "depression" is not mentioned in the full text, such as the post "I'm in trouble." I am very anxious, fearful, and depressed, and I am very grateful to the predecessors of the forum for guidance." The authors of such posts may only be netizens with relatively depressed personality, or they may be depressed patients.

\subsection{Information Sources trusted by the depression community}

In the depression community, the cognition and discussion of depression treatment are usually based on the following sources: their own illness or recovery experience, doctor's advice, social or medical news about depression, public Internet information, and patients Interpersonal communication, posts by psychological counselors in online forums. Among these, the patient's own rehabilitation experience and doctor's advice accounted for more. According to the patient's statement, it can be found that patients usually choose to trust real-life physicians and named psychological counselors in online forums.

As for the drug treatment for depression, the current medical community believes that depression must be carefully diagnosed by a physician before the symptoms and medication can be determined (Chen Junqin, 2004). It was found that in most posts related to drug treatment, patients with depression also tend to consult relevant physicians in the psychiatric department or named physicians on the Internet when choosing medications, dosages of medications, and duration of treatment.

For example, the poster "Qin" of the post "Therapeutic Knowledge of Antidepressant Drugs, Enough Treatment (Treatment Knowledge Summary)" in the depression section of the forum is a psychologist. This post first refutes the "theory of the ineffectiveness of depression drugs":

I met many friends on the Internet and they always complained that they took medicine but had no effect. One netizen even said that he had been ill for half a year and had used all antidepressants but none of them were effective. All they very resolutely told me was that drugs have no effect on depression. In fact, none of them had a full course of treatment.

In the post, the poster introduced in detail the relevant knowledge of medications for depression, the factors that cause medication failures, and the side effects of medications. In the comment area below the post, there is a comment from a depression patient netizen "C ":

Hello, I heard that you are a psychiatrist, and I have a problem with the side effects of drugs to ask you for advice.

Respondents also asked for consultation after explaining their own situation and symptoms, and the host also put forward more systematic responses and suggestions below. There are about 90 post comments in this form, all of which are questions and consultations from netizens of depression patients and responses from doctors' posters. On the 88th floor of the post, a patient with depression pointed out that seeking the advice of a doctor is very important and necessary for patients with depression:

It is necessary to consult a specialist on what kind of medicine to use and how many doses to use. It is best to be able to communicate and communicate fully. If you have any questions, tell the doctor your opinions and questions (these require "comrades in depression arms" to have relevant, Necessary medication knowledge), soliciting the opinions of doctors, and asking doctors for necessary explanations.

In the post "Ten Years! Re-diagnose! Re-treatment! Live again! "The patient netizen "sxxx001" pointed out his years of suffering from depression, and revealed that he had increased the dose of medicine on his own. In the comments of netizens below, it is clearly recommended that the host consult a professional doctor or hospital to avoid self-adjusting the use of drugs:

$S$, is the prescription prescribed by the doctor? Or call the doctor and ask (Netizen: Li Che)

Go to a specialist hospital for treatment as soon as possible. It is best not to adjust the medicine for yourself (Netizen: Tomorrow's Sun)

Do not adjust the medicine frequently, and consult a doctor to increase the dosage if necessary. (Netizen: comradein-arms)

In addition to professional doctors, patients will also seek information from other patients' posts. Since there are not many types of mainstream drugs for depression. Therefore, discussions among patients on drug efficacy, usage habits, and drug dosages are more common. For example, the post of "[Medical Information] My journey of med trial" recorded in detail the drug use experience and effects of netizens who claimed to be patients with "refractory depression patients". 
People who conduct drug tests on their bodies." There are also consultations and discussions on the efficacy of drugs for other patients with depression under this post.

It is worth mentioning that in the depression forum, patients will not only communicate the choice and efficacy of drugs but also discuss the methods and prices of depression drugs more often. Patients in depression online forums come from different regions, where prices are different, or the channels through which they choose to buy drugs are different. Patients will share the price and method of obtaining drugs with each other, or establish contacts privately, for example, in "How much do you spend per month? Reply to the post in Treating Depression:

Both the hospital and the pharmacy make half of the money. I bought it online. It turned out to be five or six hundred a month. I eat a lot. (Netizen: yu)

Why do I watch the drugs sold online are still expensive? really? On which network? (Netizen: comrade)

In the course of special therapies such as electroshock therapy, the source of information for patients with depression still mainly depends on the doctor's advice. It is worth noting that in the post "I am receiving electrotherapy..." by the netizen "LS", the patient pointed out that he had quarreled with the doctor during the electrotherapy, but still chose to trust the doctor's explanation And suggestions.

When I had the first electrotherapy, I quarreled with the doctor. I asked when it was my turn, but the doctor said that I had done it. I didn't believe it. Later, the doctor told me about the side effects of transient amnesia, and I believed to leave. Now I am in a state of amnesia, and I have lost my recent memory. The doctor said that it would take three months to recover.

Although electric shock therapy has caused more extensive discussion in online forums, in fact, there are not many depression patients receiving electric therapy. Therefore, the description of electric shock therapy in the doctor's doctor's order or media news and the latest research is still understood by depression patients. The more dominant source of this therapy.

For example, in the post "[Medical News] Latest Research Shows", the poster quoted the news that John Campbell received electric shock therapy and his depression was relieved. However, there are fewer comments and replies under such news or the latest research progress posts. Most of them are "thanks to the original poster" and "thanks for sharing", and there are fewer consultations and discussions.

\subsection{The sensitive and controversial issues in the treatment of depression}

In public forums for depression, some controversial or more sensitive topics are repeatedly mentioned, causing lively and continuous discussions. This article will make a preliminary observation and summary of several frequently occurring issues in the observed samples:

\subsubsection{Does depression need medication? Is the medication effective?}

The depression community has different opinions on whether depression requires continuous medication. Posts that believe that depression does not require medication or that medication is ineffective include the following categories:

First, after long-term drug treatment is ineffective, lose confidence in drug treatment. For example, the netizen "bright" said: After taking adequate foot treatment for two years, Leyou, Xinbaida, Ospine, Bolexin, Citalopram, and Sertraline have no effect. Said that the disease is still feeling more and more serious. This type of post does not clearly indicate that depression does not require medication, and is usually based on the self-report of the patient's own medication habits and efficacy. In the comment area, this type of post or response is more likely to resonate, such as the response of the netizen "tan": People with unsatisfactory drug effects really feel sorry for the same disease!

Second, it is believed that the drug is effective at first but gradually becomes ineffective after it develops resistance. For example, the netizen "Xiang Feng" pointed out: Me too. Some medications were effective at first, but slowly failed and relapsed after taking the medication. I think that over time, drug resistance will definitely develop. This is the limitation of drugs. In a poll conducted by 109 people, a post "[Vote] Do you think antidepressants will develop resistance? In ", $62.39 \%$ of people believe that antidepressant drugs will produce drug resistance, and some netizens under the post believe that drug resistance varies with different drug components.

The following types of posts believe that depression must be effective through medication or medication:

First, direct advice from a psychiatrist or psychologist. For example, the netizen "Consultant" clearly pointed out in the post: We can clearly tell everyone that the effectiveness of drugs on the symptoms of depression is clear, whether it is SSRIs drugs or SNRI, ...their effect is nothing Doubtful.

Second, patients who use drugs for a long time or who use drugs effectively. For example, the netizen "Sunflower", a patient who relapsed four times, mentioned: I don't believe that psychotherapy or transcranial magnetic therapy can be cured. It can only be used as an auxiliary treatment with drugs.

\subsubsection{The "mystery" of electroshock therapy}

Although there are not many users of electroshock therapy, whether this special therapy is effective and its side effects is a topic of concern in many posts on depression forums. From a diachronic point of view, electroshock therapy has gradually attracted the attention of the depression community. After a small number of patients have such a diagnosis and treatment experience, it attracts the attention of the depression community. 
Electrotherapy has a mysterious meaning in the depression community. Since there are not many patients using this therapy, individual posts mentioning that they have experience in electrotherapy will particularly attract the attention of netizens, and many netizens respond to and track follow-up situations. For example, in the post "I am receiving electrotherapy..." posted by the netizen "LS" of a patient who was asked to do ten electroshock treatments, some netizens replied the following year: I heard that the LS has completely recovered, right? It's really hard to imagine how electrotherapy is so effective, correcting our incorrect cognition?

The discussion of electric shock therapy in the community is also quite polarized. Some netizens believe that electric shock ignores the body and normal thinking of depression patients, as well as whether there are serious sequelae, this kind of treatment is very cruel. For example, in the posts of netizens "Long Skirts", the original posters rarely return to the forum for follow-up status updates. The netizen "HWI" directly pointed out in his response two and a half years later: Why did the long skirt not show up for two and a half years? A person who loves life, a person who loves sunshine, a person who hopes to heal after an electric shock, and a person who hopes that others will share their good therapeutic effects. How could it suddenly disappear in the sun after the seventh electric shock, and suddenly disappear in the sight offriends?

Some netizens believe that electrotherapy is the "last line of defense" in the treatment of depression. Some netizens even posted an article titled "Suicide or Electric Shock." Some netizens believe that electrotherapy makes them the last treatment they are willing to try when other therapies are difficult to achieve:

I have been suffering from depression for more than four months. I have tried various treatments. Many people enlightened me and cared about me. I want to go to the hospital to try electric shock therapy, maybe it is my last treatment idea (Netizen: $h x t$ )

"Moderate depression, if the doctor says no, he will be hospitalized for electrotherapy!" It seems that electrotherapy is omnipotent! "(Netizen: DB)

Electric shock treatment is a last resort! (Netizen: Wind)

Although there are not many patients trying electric shock therapy, the depression community's interest in whether electric shocks are painful, what are the sequelae, and whether they are effective can be seen in their posts and responses. Some netizens will also leave their contact information, hoping to communicate with netizens who have experienced electric shock treatment.

\subsubsection{Treatment or cure? Depression's "complete cure" theory}

This type of problem is often mentioned in the depression community, but it is often not mentioned as the subject of a post, but by the author in a post related to depression treatment. For example, the netizen "Consultant " mentioned in a post that their role is beyond doubt. However, at present, such drugs can only treat symptomatically, and it is difficult to achieve a cure effect. It means that I can control your symptoms, make you feel less uncomfortable, and let you go to work normally. The psychological counselor believes that drug therapy cannot achieve a cure, but can only control symptoms and allow patients to live a normal life. At the bottom of the post, there are patients who agree with the view that "they can't be cured completely." The netizen "Sunflower 666", who has relapsed four times, thinks: Please believe that depression can be cured. The key is to take the right medicine. In the forum, there are many cases of depression "cured" or self-identified as "self-healing". The experience of these cases seems to be evidence of the theory that depression can be completely cured. Of course, neither the author nor the netizens can tell whether the author of such a post has been cured without relapse.

In the medical world, whether depression is "treatable" or "curable", there is no clear conclusion yet. Some netizens also pointed out that there is no need to worry too much about whether it is completely good to normal, as long as the obvious symptoms of depression can be controlled.

\subsubsection{How the rumors be spread and how to clarify in the depression forum}

In the depression forum, there will also be some rumors about depression knowledge or information.

The first type of rumor is about new drugs and new treatment devices for the treatment of depression. Under such rumors or consultation posts, you can usually see the questions and responses of netizens. For example, in the post "Is the EFG Neurotransmitter Tester useful?", patients asked whether the EFG Neurotransmitter Tester can cure depression. Netizens commented below: I have seen books and documents, science and education documentaries. I have never heard of a magical instrument for comprehensive testing of neurotransmitters, nor have I heard of neurotransmitter testing that can help cure diseases. Regarding the relationship between human nerves and emotions, medical research is still in the initial stage of ignorance. (Netizen A)

The second type of rumor is often controversies about uncertainty in the treatment of depression. For example, whether the drug is effective, how many side effects of electric shock are, there is no conclusion in the medical field, but patients may post a conclusion based on their own symptoms or treatment experience. In addition to the responses of netizens, such rumors also include relevant popular science posts by doctors or psychologists for reference. For example, the netizen "Consultant " pointed out in the post: We can clearly tell everyone that the effectiveness of drugs on the symptoms of depression is clear...their effects are beyond doubt. 
In order to protect the personal information of netizens, the names of netizens in this study are anonymized or abbreviated.

\section{CONCLUSION AND DISCUSSION}

\subsection{Self-disclosure and discussion in the depression community}

The depression community has self-disclosure on the open social platform, has rehabilitation experience, vents the mood, and also has treatment information sharing and consultation. In the treatment of depression, the depression community has a considerable degree of trust in doctors, and will also capture information from the experience of named doctors or patients on the Internet. For the discussion of controversial issues, the depression community usually uses past treatment and rehabilitation experience to assist in judgment, and named physicians may play the role of clarifying rumors.

\subsection{The interaction between personal subjective experience and biomedical reality}

There is no uniform and index standard for the diagnosis and treatment of depression and the judgment of cure. Therefore, the sense of illness and cognition of the disease of depression patients are largely affected by their own disease experience. In the public posts of the depression community, the author found that patients are facing uncertain conditions or controversial issues (such as whether their own conditions can be diagnosed as depression, whether they should stop the drug, whether electric shocks can be used, etc.), to a large extent, it will be influenced by professional doctors and patients with similar experience or diagnosis and treatment experience. Professional physicians include not only hospital psychiatrists whose patients seek help in reality, but also netizens who call themselves psychiatrists or psychological counselors who are named or unnamed on the Internet.

When the patient's personal disease experience conflicts with the true scientific or biomedical information received by the patient, for example, the patient believes that he is following the doctor's advice or insisting on medication but has not improved, or the patient believes the doctor's advice, diagnosis, treatment plan, and judgment When it is inconsistent with their own disease experience, or when the patient is over-reliant on doctor's orders and medications but fails to achieve the desired psychological effect, the cognition of the depression community may fall into hesitation or doubt. Under such circumstances, according to the self-disclosure of the depression community in the forum, they usually still choose to believe the doctor's advice and medical knowledge explanation. But at the same time, their trust in so-called scientific knowledge may be reduced, and at the same time, they may be more susceptible to the influence of patient experience, celebrity experience, and social news, trying to find and try other methods of diagnosis and treatment.

In the public forum for depression, in addition to the popularization of positive medical knowledge, rumors and other bad news will inevitably appear. Due to the particularity of the causes of mental illness, its treatment methods are also very extensive, in addition to drug therapy, there are psychotherapy, physical therapy, etc. When the patient's own trust in doctor's orders is insufficient, or the ability to discriminate information on the Internet is insufficient, they may choose to believe or even practice the therapy in the forum posts, which may result in double damage to property and spirit.

Therefore, in the context of science communication to discuss diseases with special diagnostic and treatment standards such as mental illness, the author believes that how to build the trust of the patient community in biomedical knowledge and how to transmit accurate scientific and medical knowledge is a crucial topic. In the process of transferring scientific knowledge, the limitations of science and medicine and the uncertainty in the treatment process should also be included, so that the self-awareness and disease cognition of the patient community can be more accurate.

\subsection{Opportunities and Challenges of Healthy Communication in the Internet Information Age}

The online community for depression patients is both a "web of information" and a "web of mutual assistance." The patient's health information acquisition model has changed from vertical reception to horizontal interconnection, which not only enhances the scientific understanding of diseases but also enhances the sense of psychological security in interpersonal interaction. Forum managers and forum editors play the role of "goalkeepers," while named physicians and popular netizens in the forum act as opinion leaders. Netizens have changed from passive audiences to active questioners, disseminators, and issue setters. The decentralized online discourse space returns the subjectivity of the audience, and communication and interaction that is not restricted by time and space give ordinary citizens greater initiative.

However, there are rumors, unverified prescriptions, and panic that spread at the same time as health knowledge. Over the past two decades, as medical knowledge has been updated and improved, some rumors have been identified and clarified. But patients' uncertainty about disease treatment has remained consistent. There is a wide range of treatments for depression, and treatment options vary from person to person. In addition to warming each other in the online community, patients may also "infect" panic. They may doubt their own treatment plan due to the negative diagnosis and treatment experience of others, and may even reduce their willingness to seek medical treatment. Therefore, when thinking about how to popularize medical knowledge and build the trust of the patient community in authoritative medical knowledge, the limitations, uncertainties, and the particularities of diseases and their treatment should also be taken into consideration. 
Interconnection is a communication trend in the Web 2.0 era. New media that are decentralized, highly interactive, real-time efficient, and not limited by time and space bring new vitality to the popularization of health information. In this new type of risk communication mode, how to establish a new fact-checking mechanism and establish ordinary people's trust in scientific and medical knowledge are new challenges. How to respect the subjectivity of the audience, stimulate the initiative of ordinary people to learn risk knowledge, and at the same time appropriately guide the public rationality, is a new topic of healthy communication.

\section{RESEARCH LIMITATIONS AND FUTURE DEVELOPMENT DIRECTIONS}

First, the samples observed in this study are limited, so we can only make rough observations and make simple classifications. It is difficult to make deeper attributions or draw more general conclusions. If there is sufficient time in the future, the scope and number of research samples can be further expanded and more universal research results can be obtained.

Second, the time span of the samples observed in this study is relatively long, and some posts were posted 15 to 20 years ago. During this period, the treatment of depression has also developed and progressed to a certain extent. Due to the time limit of depression research, the historical dimension of depression treatment is not yet perfect, and it can be continuously compensated in the future.

\section{REFERENCES}

[1] Abrams, R. (2002). Electroconvulsive therapy. Oxford University Press.

[2] American Psychiatric Association (2013).Diagnostic and statistical manual of mental disorders, 5th edition: DSM-5. Washington, DC: Author.

[3] Chen Caihui, Xia Yixin, Zhou Guiru (2004). Clinical Analysis of Cognitive Behavior Group Therapy in Patients with Depression . Tzu Chi Journal of Nursing, 3(1), 19-27.

[4] Chen Junqin (2004). Depression and Depression . Taipei: Healthy.

[5] Dong Wei (2010). Research on Depression Reporting from the Perspective of Health Communication . News World , (5), 91-93.

[6] Freud (2018). Introduction to Psychoanalysis (New Edition) (Vol. 281). Left bank.

[7] Jiang Yang (2018). Research on the Communication of Depression Issues from the Perspective of Health Communication , Master's thesis. Chongqing: Chongqing University.

[8] Klin, A. \& Lemish, D. (2008). Mental disorders stigma in the media: Review of studies on production, content, and influences. Journal of Health Communication, 13(5), 434-449.

[9] Lin Meiyang (2001), Study on the Impact of Cognitive Behavioral Therapy Group on Patients with Depression and the Treatment Effect, unpublished doctoral dissertation. Changhua: National Changhua Normal University.

[10] Liu Yi'an (2017). Is Electrotherapy Scary to Treat Depression?

[11] Morris, G. (2006). Mental health issues and the media- An introduction for health professionals. New York: Routledge.

[12] Nairn, R. G.. (2007). Media portrayals of mental illness, or is it madness? A review. Australian Psychologist, 42(2), $138-146$.

[13] Pang Xu (2016). Research on Depression Reporting from the Perspective of Health Communication (2011-2015) Master thesis of Journalism of East China Normal University.

[14] Philo, G.. (Ed.) (1996). Media and mental distress. London and New York: Longman.

[15] Ren Jinzhou, Kang Yunkai (2015). Depression from the Perspective of Health Communication in my country's Television Media, Media Today, 23(11), 165-167.

[16] Shu Manqiao, Xu Weilong, Anbang, Zhao Fangxia, Zhang Juan (2014). The Effect of Psychotherapy on the Rehabilitation of Depressed Patients. Occupational Health and Injuries , 29(2), 162-163.

[17] Stansbury,K., \&Gunnar, M. R. (1994). Adrenocortical activity and emotion regulation. Monographs of the Society for Research in Child Development, 59(2-3), 108-134.

[18] Wahl, F. O., \& Roth, R. (1982). Television images of mental illness: Results of a metropolitan Washington media watch. Journal of Broadcasting, 26(2), 599-605.

[19] Wahl, F. O., Wood, A., \& Richards, R. (2002). Newspaper coverage of mental illness: Is it changing? Psychiatric Rehabilitation Skills, 6(1), 9-31.

[20] Wu Mengjin, Xu Meiling (2011). The Biomedical Reality, Social Cultural Reality and News Reappearance of Depression: Taking Taiwan's China Times and Apple Daily as examples, Journal of Communication and Sociology, (17), 87-121.

[21] Ye Suijun (2010). Eight weeks of exercise intervention on the physical activity, physical fitness and quality of life of the visually impaired. Dissertation of Pingtung University of Science and Technology Department of Leisure Sports and Health Care. 